\title{
Pseudoaneurisma de la fibrosa mitroaórtica: un hallazgo de una complicación tardía
}

\author{
Ciro Barrantes* 1,a; Johan Fernández ${ }^{1, b}$
}

RESUMEN

Se presenta el caso de un varón de 46 años, asintomático y con antecedente de prótesis mecánica aórtica monodisco desde hace 25 años. Mediante el empleo de la ecocardiografía transtorácica, ecocardiografía transesofágica y tomografía cardiaca, se le diagnostica, incidentalmente, un pseudoaneurisma de la fibrosa intervalvular mitroaórtica (región de tejido fibroso localizada entre el velo anterior de la válvula mitral y los velos aórticos izquierdo y no coronariano). Debido al tamaño del pseudoaneurisma, se decide realizar una cirugía. Este artículo muestra la presentación tardía de un pseudoaneurisma en esta localización que aparece como una complicación del reemplazo de la válvula aórtica por endocarditis. Se destaca la evolución asintomática, el tamaño y la cirugía que preserve la válvula cuando no está comprometida. Las diferentes técnicas de imagen son complementarias y ayudan en tomar las decisiones del caso.

Palabras clave: Endocarditis; Pseudoaneurisma; Válvula aórtica (Fuente: DeCS BIREME).

\section{Pseudoaneurysm of the mitral-aortic intervalvular fibrosa: a discovery of a late complication}

\section{ABSTRACT}

This is the case of a 46-year-old man, asymptomatic, with a history of single-disk mechanical aortic prosthesis implanted 25 years ago, who was incidentally diagnosed with a pseudoaneurysm of the mitral-aortic intervalvular fibrosa (a region of fibrous tissue located between the anterior leaflet of the mitral valve and the left and non-coronary aortic leaflets) discovered by means of a transthoracic echocardiography, transesophageal echocardiography and cardiac computed tomography. Surgery was decided because of the size of the pseudoaneurysm. This article shows the late presentation of a pseudoaneurysm in this location caused by a complication of an aortic valve replacement due to endocarditis. It also highlights the asymptomatic evolution, the size of the pseudoaneurysm and the election of a valve preserving surgery because the valve was not compromised. The different imaging techniques are complementary to the research and help to make the pertinent decisions.

Keywords: Endocarditis; Aneurysm, false; Aortic valve (Source: MeSH NLM).

1 Hospital Nacional Guillermo Almenara Irigoyen-EsSalud. Lima, Perú.

a Médico Cardiólogo.

b Médico Cirujano Cardiovascular.

*Autor corresponsal. 


\section{INTRODUCCIÓN}

La endocarditis tiene una incidencia baja: alrededor de tres a diez casos por 100000 habitantes, y del $20 \%$ al $50 \%$ de los pacientes desarrollan complicaciones que requieren cirugía ${ }^{(1,2)}$. El pseudoaneurisma se define como la ruptura de pared no transmural, es decir, no se dañan todas sus capas, la adventicia y el pericardio circundante se conservan indemnes. Los pseudoaneurismas son una complicación rara pero grave de la endocarditis. La extensión perivalvular incluye la formación de abscesos, pseudoaneurismas o fístulas. La fibrosa intervalvular mitroaórtica (FIMA) es una región compuesta de tejido fibroso localizada entre el velo anterior de la válvula mitral y los velos aórticos izquierdos y no coronariano. Es la localización más frecuente de la extensión perivalvular en endocarditis aórtica (3). Cuando ocurre una extensión perivalvular es más difícil el control de la infección lo que conlleva a un pobre pronóstico e incrementa la posibilidad de necesitar cirugía. Existen pocos reportes publicados de esta enfermedad, por lo que la información sobre su diagnóstico y tratamiento es escasa. En este artículo se revisan los trabajos publicados obre este cuadro y se destaca la importancia del diagnóstico y tratamiento adecuados del pseudoaneurisma de la FIMA. Para esta investigación se cumplieron los principios éticos protección de la identidad del paciente.

\section{CASO CLÍNICO}

Paciente varón de 46 años. Hace 25 años presentó endocarditis y se reemplazó la válvula aórtica con una prótesis aórtica monodisco. Tiene antecedentes de hipertensión arterial y diabetes mellitus en tratamiento. Hace dos años acude a control rutinario al Servicio de Cardiología y no refiere molestias. En el examen físico solamente se detecta un soplo sistólico eyectivo en foco aórtico. Se le realiza una ecocardiografía transtorácica (ETT), que informa de una prótesis aórtica mecánica, que funciona normalmente, e insuficiencia mitral leve, sin otro hallazgo relevante. Continúa el seguimiento clínico y permanece asintomático. Hace seis meses, se le realiza una ETT de control y en la vista de cuatro cámaras se encuentra un saco hipoecogénico de paredes irregulares, pulsátil y no muy bien definido (Figura $1 \mathrm{~A}$ ): a la izquierda, señalado con flecha roja). Luego, se realiza una ecocardiografía transesofágica (ETE) y se observa un saco pulsátil, que se expande en sístole y colapsa en diástole, de bordes irregulares, aparentemente tabicado, adyacente y por encima del tracto de salida del ventrículo izquierdo (imágenes ortogonales, a $30^{\circ}$ y $120^{\circ}$, figuras $1 \mathrm{~B}$ y $1 \mathrm{C}$, respectivamente)
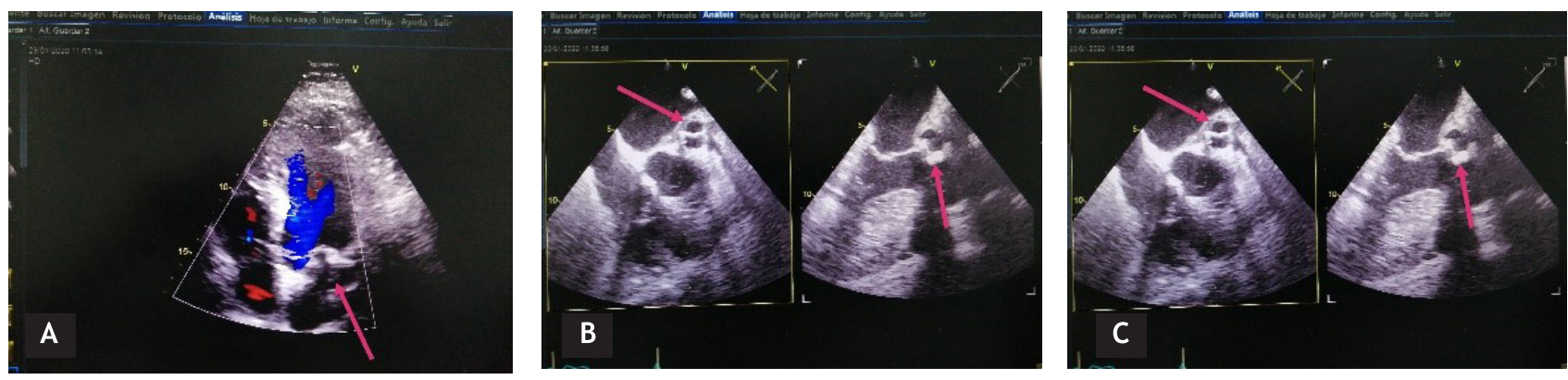

Figura 1. (A) ETT a la izquierda, (B) ETE imagen ortogonal a $30^{\circ}$ y (C) ETE imagen ortogonal a $120^{\circ}$

La figura 2A (flecha blanca) muestra el orificio de entrada de cuello estrecho y flujo sistólico de alta velocidad, definido como pseudoaneurisma. En el ETE 3D (2C) se destaca la

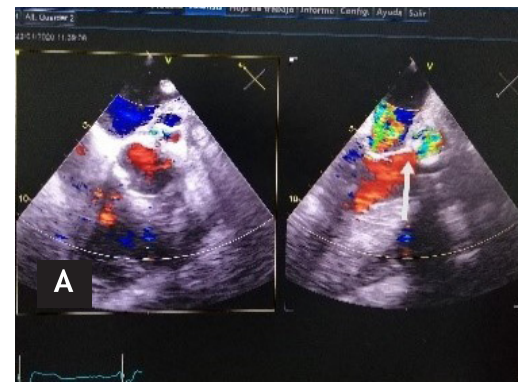

Figura 2. (A) Ecotransesofágico 2D y (B) ecotransesofágico 3D relación entre el tracto de salida del ventrículo izquierdo (flecha azul) y el pseudoaneurisma (flecha roja).

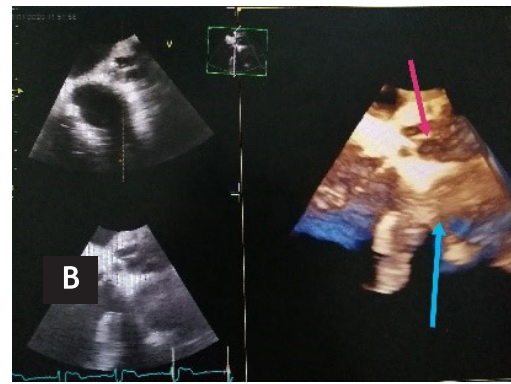


En la tomografía cardiaca con reconstrucción 3D (Figura 3, flecha roja) se observa un saco de paredes irregulares de 10 x $8 \mathrm{~cm}$ que se origina por debajo de la prótesis aórtica, se destaca su relación con estructuras adyacentes y su tamaño. También se evidencia que no hay compresión de la coronaria izquierda. Ante el riesgo de ruptura del pseudoneurisma debido a su tamaño, la junta médica decide realizar una intervención quirúrgica el 18 de febrero del 2020 que consistió en una esternotomía, canulación, aortotomía y también se confirmó la competencia de la válvula aortica monodisco. El pseudoaneurisma fue abierto y encontraron un orificio por el que pasa una tijera como tutor, luego se comprueba la comunicación entre el pseudoaneurisma y el tracto de salida del ventrículo izquierdo.

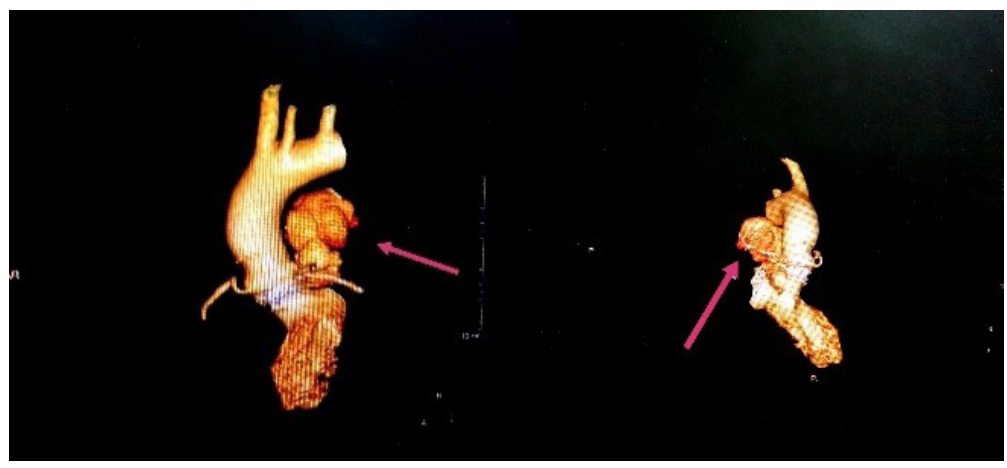

Figura 3. Tomografía cardiaca con reconstrucción 3D

Con la válvula cerrada, se introduce la tijera por el orificio del pseudoaneurisma (Figura 4A) y luego, con la válvula abierta la punta de la tijera se puede observar en el tracto de salida del ventrículo izquierdo (Figura 4B). El orificio de entrada se cierra con un parche pericárdico y la válvula se preserva. El paciente evoluciona favorablemente y es dado de alta a los seis días, permanece asintomático en el último control dos meses luego de cirugía.

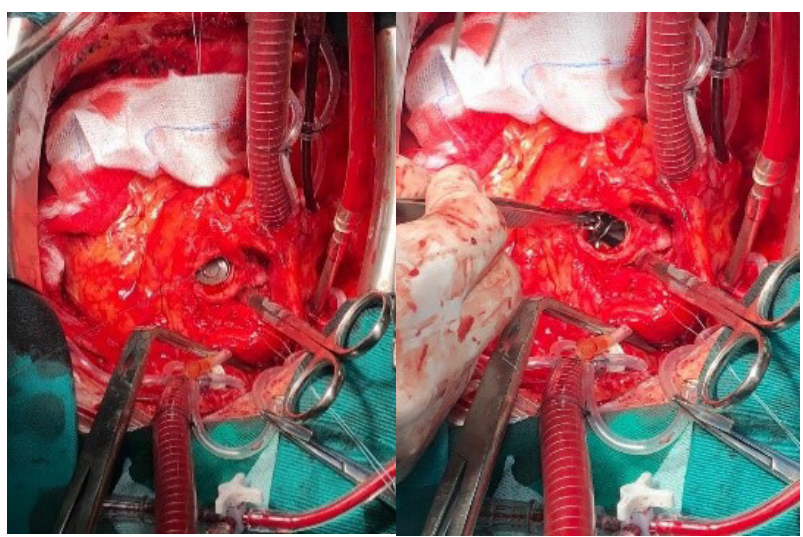

Figura 4. (A) y (B) Cirugía cardiaca

\section{DISCUSIÓN}

Los pseudoaneurismas cardiacos aparecen en diferentes localizaciones y obedecen a diferentes etiologías. Pueden ser agudos (inestables, con alto riesgo de ruptura) o crónicos (se diagnostican al complicarse o incidentalmente).

El pseudoaneurisma se diferencia del aneurisma por la localización, flujo, tamaño del cuello y número de capas comprometidas: el aneurisma usualmente es apical, la apertura o cuello es mayor al $50 \%$ del diámetro del pseudoaneurisma, las paredes están constituidas por tejido fibroso y miocardio, y no tiene flujo de alta velocidad, mientras el pseudoaneurisma es, generalmente, posterolateral, de cuello estrecho y flujo de alta velocidad. Por otro lado, el absceso es más pequeño, no pulsátil y no muestra flujo.

El pseudoaneurisma de la fibrosa intervalvular mitroaórtica (FIMA) puede formarse después de un infarto agudo de miocardio, por un trauma torácico, luego de manipular la zona durante la cirugía cardiaca, por endocarditis infecciosa, o puede ser congénito. La FIMA es una zona de baja irrigación y susceptible de contraer una infección 
que puede llegar por contigüidad con la pared aórtica o por diseminación a través del jet de regurgitación. En el presente caso clínico, la diseminación pudo ocurrir durante el episodio de endocarditis y debilitar la pared, que puede romperse en la fase activa o después de algún tiempo, y formarse el pseudoaneurisma, que puede crecer súbita o lentamente como en este paciente.

Las complicaciones de los pseudoaneurismas son la ruptura hacia el pericardio (y producir taponamiento cardiaco), la compresión de estructuras cardiacas (como la arteria coronaria izquierda) o fistulización hacia las aurículas, y embolismos por la formación de coágulos dentro de la cavidad del pseudoaneurisma. También puede producir compresión sistólica de la válvula mitral y originar insuficiencia mitral severa. La presentación clínica puede ser con insuficiencia cardiaca, dolor torácico, disnea, hemoptisis, muerte súbita, y embolismos sistémicos o cerebrovasculares. Puede comprimir la arteria coronaria izquierda y causar isquemia ${ }^{(4)}$. En el $12 \%$ de los casos, el paciente se encuentra asintomático, pero el pseudoaneurisma aparece como una masa pulsátil con expansión en sístole y colapso diastólico.

Los principales factores de riesgo asociados a la formación de un pseudoaneurisma de la FIMA son la endocarditis infecciosa y la prótesis aórtica. Otros factores son trauma quirúrgico, insuficiencia aórtica, aorta bicúspide, otros procedimientos quirúrgicos como reemplazo de raíz aórtica o intervencionistas como cateterismo cardiaco, ablación con radiofrecuencia, cierre de comunicación interventricular (CIV), comunicación interauricular (CIA), persistencia del conducto arterioso (PCA), coartación de la aorta, infección por estafilococo coagulasa-negativo, arteritis de Takayasu o anomalía congénita ${ }^{(3,5)}$.

El diagnóstico se realiza por ecocardiograma, cateterismo cardiaco, tomografía o resonancia magnética. En nuestro paciente, la ETT era dudosa, pero con la ETE se pudo determinar la presencia del pseudoaneurisma (a $130^{\circ}$ como un saco en la región superior del seno valsalva no coronariano, con flujo de alta velocidad y comportamiento dinámico, que se expandía en la sístole y se colapsaba en la diástole) así como el punto de entrada, y con la tomografía cardiaca se precisó la anatomía exacta, el tamaño y su relación con las estructuras adyacentes. La ETE también ayuda durante el momento intraoperatorio. La ETE en tercera dimensión (3D) proporciona información anatómica y morfológica del pseudoaneurisma, las relaciones con otras estructuras, información que es importante para el diagnóstico y manejo en sala de operaciones ${ }^{(6)}$.

En relación al tratamiento se considera al intervencionismo ${ }^{(7,8)}$, la cirugía ${ }^{(9)}$ y, en algunos, casos solo el seguimiento con ecocardiografía o algún otro método de imágenes cada 6 a 12 meses (en el caso de los pseudoaneurismas pequeños y asintomáticos) ${ }^{(10,11)}$.
Sahan et al. (2015) revisaron los casos publicados y encontraron 166 pacientes con pseudoaneurisma de la FIMA. Sus hallazgos principales fueron que en la mayoría de los casos se presentó una infección asociada (endocarditis activa o pasada) o un trauma quirúrgico, y que el tratamiento recomendado fue una cirugía ${ }^{(5)}$.

En 2016, Han J. et al. encontraron resultados similares en una serie de nueve casos. Ocho pacientes tenían endocarditis activa; tres, se complicaron con una fístula, ocho fueron operados y uno, por el alto riesgo quirúrgico, tuvo solamente seguimiento clínico ${ }^{(6)}$.

La cirugía debe planearse cuidadosamente. Se debe tener en cuenta el acceso al pseudoaneurisma, la estrategia de canulación y la realización o no de paro circulatorio hipotérmico. En nuestro caso la técnica quirúrgica consistió en reesternotomía, puesto que se encontró, por tomografía cardiaca, una adecuada distancia entre la tabla inferior del esternón y el pseudoaneurisma. La válvula era competente, por lo que no se realizó un cambio valvular y se cerró el pseudoaneurisma con parche pericárdico en el orificio de entrada.

Cada caso debe individualizarse y decidir el tratamiento de acuerdo a diversos factores como la forma de presentación (aguda o crónica), debe intervenirse si el tiempo de enfermedad es menor de tres meses y posterior a un infarto, si crece rápidamente o causa síntomas como taquiarritmias, dolor torácico, falla cardiaca, embolismo, isquemia, o por el tamaño (riesgo de ruptura) ${ }^{(12)}$.

La identificación y el tratamiento precoz de una endocarditis infecciosa con extensión perivalvular deben realizarse para erradicar la extensión de la infección durante la cirugía y evitar complicaciones posteriores tan graves como la formación de un pseudoaneurisma (13).

Se puede evidenciar el compromiso precoz de la FIMA en el momento del diagnóstico de endocarditis y planear la limpieza de la zona durante el cambio de válvula aórtica para evitar esta complicación. Un trabajo en pacientes sanos encontró que un grosor de FIMA mayor de $6 \mathrm{~mm}$, por ETE, puede ser un signo temprano de difusión perivalvular y hacer sospechar de la formación incipiente de un absceso periaórtico, que puede terminar en un pseudoaneurisma ${ }^{(14)}$.

Este artículo destaca la presentación tardía de un pseudoaneurisma de la FIMA como la complicación de un reemplazo de válvula aórtica por endocarditis y su evolución asintomática por muchos años. Se decidió la cirugía por el tamaño del pseudoaneurisma y se preservó la válvula al no estar comprometida. Las técnicas de imagen (ETT, ETE $2 D$ y $3 \mathrm{D}$, y tomografía cardiaca) son complementarias y juegan un rol importante en el diagnóstico y tratamiento 
adecuados del pseudoaneurisma de la FIMA.

Contribuciones de los autores: CB y JF participaron en la concepción, recolección, redacción, interpretación de los datos y revisión crítica del manuscrito.

Conflicto de interés: Los autores declaran no tener conflictos de interés.

Fuentes de financiamiento: El artículo fue financiado por los autores.

\section{REFERENCIAS BIBLIOGRÁFICAS}

1. Bonow R, Mann D, Zipies D, Libby P, Braunwald E. Tratado de Cardiología de Braunwald (Volumen $\mathrm{N}^{\circ}$. 1). 9na ed. Barcelona: Elsevier; 2013

2. Yang E, Frazee B. Infective Endocarditis. Emerg Med Clin North Am. 2018; 36(4): 645-63.

3. Habib G, Lancellottti P, Antunes M, Bongiorni M, Casalta JP, Del Zotti F, et al. 2015 ESC Guidelines for the management or infective endocarditis. Eur Heart J. 2015; 36: 3075-123.

4. Acioli Pereira L, Fontes P, Alcantara J, Palandri AC, Renato E, Bento LC. Pseudoaneurisma gigante de tracto de salida de ventrículo izquierdo: una patología rara. Rev Port Cardiol. 2013; 32(6): 541-4.

5. Sahan E, Gül M, Sahan S, Sokmen E, Guray YA, Tufekcioglu 0. Pseudoaneurisma de la fibrosa intervalvular mitro-aórtica: una nueva revisión exhaustiva. Herz. 2015; 40(2): 182-9.

6. Han J, He Y, Gu X, Sun L, Zhao Y, Liu W, et al. Echocardiographic diagnosis and outcome of pseudoaneurysm of the mitral-aortic intervalvular fibrosa. Medicine. 2016; 95(11): e3116.

7. Jimenez S, Garcia E, Gonzáles A, Delcán J. Cierre percutáneo de seudoaneurisma de la fibrosa mitroaórtica. Rev Esp Cardiol. 2005; 58(12): 1473-5.

8. Zanuttini D, Garcia E, Lazarte J, Costantini C, Tabine S, Freitas M, et al. Tratamento percutáneo de pseudoaneurisma da zona fibrosa intervalvar mitroaórtica. Rev Bras Cardiol Invasiva. 2011; 19(3): 327-31.

9. Rotta A, Dueñas R, Rodriguez R, Martorell C. Pseudoaneurisma de la fibrosa mitro-aórtica en endocarditis de aorta bicúspide: a propósito de un caso. Rev Med Hered. 2018; 29(3): 168-72.

10. Salerno D, Donati G, Forconi S, Gori T. Pseudoaneurisma gigante de fibrosa mitro-aórtica: diagnóstico incidental. Intern Emerg Med. 2008; 3: 279-82.

11. Rosario Chieppa DR, Scardigno AD, Deluca G, Capodivento S, Quinto $N$, Cicala M. Conservative treatment of a pseudoaneurysm of the mitral-aortic intervalvular fibrosa: 9 years of follow-up. G Ital Cardiol (Rome). 2017; 18(6): 525-8.

12. Muretti M, Elmahdy W, Ttofi I, Mozalbat D, Murphy M, Asimakopoulos G. Reparo quirúrgico de pseudoaneurisma de la fibrosa mitro-aórtica en un paciente 13 años después de reemplazo de raíz aórtica. Tex Heart Inst J. 2019; 46(2): 147-50.

13. Gotor-Pérez C, López-Gude M, Ceneteno-Rodriguez J, Perez de la Sota E, Eixerés-Esteve A, Cortina-Romero J. Pseudoaneurisma en tracto de salida de ventrículo izquierdo. Complicación por endocarditis infecciosa en paciente intervenido previamente de cirugía de raíz aórtica. Cir Cardiov. 2019; 26(3): 172-4.

14. Cresti A, Baratta P, De Sensi F, Solari M, Sposato B, Minelli S, et al. Valores normales del grosor de la fibrosa intervalvular mitro-aórtica: un estudio multimodal. J Cardiovasc Echography. 2019; 29: 95-102.

\section{Correspondencia: \\ Ciro Barrantes}

Dirección: Av. Grau 800, La Victoria. Lima, Perú.

Teléfono: 941392086

Correo electrónico: cirobarrantes@yahoo.com

Recibido: 17 de abril de 2020

Evaluado: 09 de agosto de 2020

Aprobado: 11 de agosto de 2020

(c) La revista. Publicado por Universidad de San Martín de Porres, Perú. (cc) $\mathbf{B Y}$ Licencia de Creative Commons Artículo en acceso abierto bajo términos de Licencia Creative Commons Atribución 4.0 Internacional. (http://creativecommons.org/licenses/by/4.0/)

ORCID iDs

Ciro Barrantes Alarcon $\quad$ https://orcid.org/0000-0003-3126-6226

Johan Fernández $\quad$ https://orcid.org/0000-0001-5888-4185 